\title{
Attachment in Children with ADHD
}

\author{
Sebastian Franke1, Ruediger Kissgen1, Maya Krischer², Kathrin Sevecke ${ }^{3}$ \\ ${ }^{1}$ Faculty II, Developmental Science and Special Education, University of Siegen, Siegen, Germany \\ ${ }^{2}$ Clinic of Child and Adolescent Psychiatry and Psychotherapy, University of Cologne, Cologne, Germany \\ ${ }^{3}$ Department of Child and Adolescent Psychiatry, Medical University of Innsbruck, Innsbruck, Austria \\ Email: sebastian.franke@uni-siegen.de
}

How to cite this paper: Franke, S., Kissgen, R., Krischer, M. and Sevecke, K. (2017) Attachment in Children with ADHD. Journal of Behavioral and Brain Science, 7, 497-510.

https://doi.org/10.4236/jbbs.2017.711035

Received: September 29, 2017

Accepted: November 5, 2017

Published: November 8, 2017

Copyright $\odot 2017$ by authors and Scientific Research Publishing Inc. This work is licensed under the Creative Commons Attribution International License (CC BY 4.0).

http://creativecommons.org/licenses/by/4.0/

(c) (i) Open Access

\begin{abstract}
Objective: Parallels in findings of attachment and ADHD research suggest a connection between both constructs. However, the few articles dealing with that tie investigate children with all conceivable expressions of ADHD and have not found sufficient evidence regarding the connection between ADHD and attachment. This study sought to collect evidence of a possible connection between the two aspects among children with the predominantly inattentive ADHD type. Method: The sample consisted of 93 children aged 5 - 9 years, 48 of whom had an ADHD diagnosis of the predominantly inattentive type. Attachment was coded using the German adaptation of the Attachment Story Completion Task, externalizing behavior problems were rated using the Child Behavior Checklist 4 - 18. ADHD was assessed using both clinical diagnoses as well as the German ADHD Rating scale. Results: Results revealed a large difference in the distribution of attachment classification between children with ADHD and those without ADHD. Regression analysis, however, showed no independent effect on ADHD when externalizing behavior problems were controlled for. Conclusions: Both constructs seem to be connected, however, in the investigation of the influence of attachment on the disorder, ADHD as a global construct is too unspecific. As opposed to previous studies, considering only the inattentive type, the relation might completely be moderated by externalizing behavior problems. Subsequent studies should first focus on specific symptom patterns which at best should be analyzed within longitudinal studies.
\end{abstract}

\section{Keywords}

Attachment, ADHD, Externalizing Behavior Problems, School Children

\section{Introduction}

Research seeking to explore the connection between attachment and symptoms of ADHD is very limited. Although some suggestive evidences have been ga- 
thered between the two, the tie between ADHD and attachment is far from being proven. This is due to at least two reasons: 1) A theoretical connection between both constructs can be expected, because there are quite a few parallels between findings from attachment research and the core symptoms of ADHD [1]. 2) The consideration of the construct ADHD as a general and comprehensive disorder hinders an investigation of the specific ties that might exist within certain behavioral ranges.

\subsection{Parallels in Research of Attachment and ADHD}

Children with ADHD present a major challenge to everyday family life, from their first years on. Behaviors associated with all three major symptom categories of ADHD-inattention, hyperactivity and impulsivity-interfere with the primary caregiver's endeavor in approaching the children's signals in a sensitive way. At the same time, sensitivity in dealing with childhood signals ("a mother's ability to perceive and interpret accurately her infant's signals and communications and then respond appropriately" [2]) is crucial for a child in forming a secure attachment with the primary caregiver up to their first year. Guttmann-Steinmetz, Crowell, Doron, \& Mikulincer [3] have found an association between the secure base scripts of mothers-their mental representations of attachment-and their schoolchildren in a sample of children without ADHD. In a sample of children with ADHD, this association disappears. Thus, the preconditions for developing a secure attachment appear to be less favorable for children with ADHD because their parents are facing challenges that impair their ability for providing a secure base, i.e. their emotional and physical availability that facilitates the child's exploration of the physical environment.

In addition, research shows that insecurely attached children are more susceptible to problems with behavior and emotional regulation [4]. Similarly, problems of self-regulation such as inhibition, impulse control, patience or perseverance are pivotal elements of ADHD and some researchers even conceptualize it as a disorder of self-regulation [5] [6] [7]. This shared connection might lead to the assumption that early child-parent interactions may play a role in the impairment of self-regulation in children with ADHD [6] [8].

Moreover, literature suggests a positive influence of attachment security on certain domains of competencies or skills that are impaired in children with ADHD [9]. In comparison to insecure attachment, secure attachment is associated with greater enthusiasm, more willingness to cooperate, extended perseverance as well as a greater effectiveness [10]. Enhanced performance in attention-related tasks and an increased attention span could likewise be linked to secure attachment [11] [12]. Other studies found an association between attachment security in early childhood and cognitive impulse control, delay of gratification and task orientation at the age of six years [13] [14]. Again, these findings solely depict parallels between two subjects of research; however, because of their similarity, it seems reasonable to assume an interrelation between 
certain aspects of both constructs.

\subsection{The Concept of ADHD in Attachment Related Research}

Results from research dealing with both ADHD and attachment are heterogeneous because very rarely the concept of ADHD is examined in detail. The term attention deficit hyperactivity disorder has become a collective term for pathological peculiarities that appear within a wide range of behavior problems [15]. Previous research on attachment and ADHD conceptualizes ADHD as a general construct and does not specify the various phenotypic expressions that are in the focus of interest.

The International Statistical Classification of Diseases and Related Health Problems, ICD-10 [16] distinguishes a simple disturbance of activity and attention (F90.0) from hyperkinetic conduct disorders (F90.1), as well as from other or unspecified hyperkinetic disorders (F90.8 and F90.9). These classifications are based on the main characteristics inattention, hyperactivity and impulsivity. Yet, a separate category (F90.1) is assigned to children that show particularly deviant types of interaction with their social environment. Thus, the behavioral aspects that are subsumed under the global construct of ADHD may be expressed through reduced attention and/or increased nervousness on the one hand or dissocial acts of interaction on the other hand. These two extremes do by no means cover the whole range of possible behaviors within that scope, but show the heterogeneity of the behavioral spectrum.

The equally common Diagnostic and Statistical Manual of Mental Disorders, DSM-5 [17], valid since May 2013, contains only a few new features of this disorder compared to its predecessor DSM-IV TR [18]. While the symptom categories inattention and hyperactivity/impulsivity are maintained as well, the various sub-types within the DSM-5 are now understood as presentations that may be changeable during the lifespan. Specific descriptions are presented and examples of individual criteria are cited. Besides, ADHD can also be diagnosed later in life and is integrated in the classification of neurodevelopmental disorders to account for correlates between ADHD and brain development. Again, this coding system clearly demonstrates that a wide range of different symptoms is summarized within the entire picture of ADHD. While the new features within the diagnosis criteria of the DSM-5 incorporate a multifactorial genesis of ADHD, they still do not allow for any new assumptions about a specific etiology. Therefore, it is the responsibility of the researcher to consider the particular expressions of this disorder in a differentiated manner.

\subsection{Attachment \& ADHD}

Of all attachment qualities, disorganized attachment in early childhood is the subtype that most clearly indicates a connection with later psychopathology or abnormality [19] [20]. Not surprisingly, the studies combining attachment and ADHD in children most notably find a link between disorganized attachment 
and ADHD [21] [22] [23] [24]. Furthermore, one study also analyzed attachment representations in mothers of children with and without ADHD [25]. The distributions differed greatly from one another and the insecure representations as well as disorganized attachment was found far more often in the group of mothers whose children had the diagnosis ADHD compared to mothers of children without ADHD.

Pinto et al. [22] showed in a non-clinical sample that disorganized attachment in infancy was associated with symptoms of inattention and hyperactivity at age seven. Goldwyn et al. [26] showed that disorganization was related to the attention problem subscale of the Child Behavior Checklist (CBCL, [27]) but not to aggression or rule-breaking behaviors [23].

Thorell et al. [24] found a relation between disorganized attachment and ADHD symptoms as these were assessed one year after the rating of the attachment representations. In a sample of $81 / 2$ year old children $(\mathrm{N}=100)$, externalizing behavior problems were also measured, using the Children's Behavior Questionnaire by Rutter, Tizard and Whitmore [28], as well as executive functioning (response inhibition, working memory). Even after controlling these two variables, the association between disorganized attachment and ADHD symptoms persisted. This finding indicates two things: 1) regarding cognitive functioning, these results show that it is the specific ADHD symptoms that are associated with disorganized attachment rather than cognitive deficits, which might occur in the course of ADHD; 2) according to the authors, there is a strong overlap between externalizing behavior problems and ADHD [18] [29], yet factor analyses confirmed a conceptual difference between those two constructs [30]. Thus, controlling for externalizing behavior problems is essential when investigating the association between attachment and ADHD. Comparable results are shown by Bohlin et al. [21] using the same methodology, as well as Scholtens et al. [23].

The heterogeneity of the samples used in research so far allows only poorly differentiated and inconsistent assertions about the relationship to other constructs. Instead, individual aspects of the disorder should first be considered separately in order to link specific symptom classes with respective constructs. The present study considers only those children whose disorder is based on the main aspects of inattention, hyperactivity and impulsivity and who do not in addition display a conduct disorder. The children who, in addition, display a characteristic phenotype through impulsivity, and to whom a hyperkinetic conduct disorder has been attributed, were not subject to our investigation. Their symptoms differ, sometimes substantially, from children whose diagnosis has been made without the presence of impulsivity, and they therefore lack phenotypic comparability because they may manifest in entirely different behavioral spectra.

\subsection{Aim of the Present Study}

1) The first aim of this study is to compare the distributions of attachment representations between children with an ADHD diagnosis and children in an un- 
affected control group. It is assumed that children with ADHD less often exhibit a secure attachment representation and, moreover, that in this group disorganized attachment is more frequently represented.

2) The relationship between ADHD and both attachment insecurity and disorganized attachment is analyzed, controlling for externalizing behavior problems. This relationship is investigated using a regression model with ADHD as the dependent variable. Both attachment and externalizing behavior problems serve as predictors to control their influence.

\section{Methods}

\subsection{Study Participants}

In a research collaboration between the Department of Special Education and Rehabilitation within the Faculty of Human Science and the Polyclinic for Psychiatry and Child and Adolescent Psychotherapy at the University of Cologne, 93 children between the ages of 5 and 9 years $(M=7.32$; $S D=0.84)$ were assessed over a period of two years (Table 1 ). The sample consisted of 48 subjects with ADHD who were all under hospital treatment at the Clinic of Child and Adolescent Psychiatry and Psychotherapy at the University of Cologne during that time (clinical population); the group of unaffected subjects comprised 45 children, drawn for the general population. The sample size resulted from the children being available for assessment during that period. Only children with the ICD-10 diagnosis F90.0 (disturbance of activity and attention) or F90.8 (other hyperkinetic disorders) or F90.9 (hyperkinetic disorder, unspecified) were included in the study. With 49 girls and 44 boys the gender distribution can be viewed as balanced. The study was conducted concordant to the prevalent ethical standards, using only non-invasive measures.

\subsection{Clinical Diagnoses}

For each case, the clinical diagnoses were made in consultation with the relevant senior physician via the junior physician of the treatment unit.

\subsection{Story Completion Procedure in Doll Play (SCPDP)}

SCPDP [31] is the German adaptation of the Attachment Story Completion Task

Table 1. Description of the sample.

\begin{tabular}{cccc}
\hline & \multicolumn{2}{c}{ Diagnosis } & Total \\
\cline { 2 - 3 } & ADHD & No ADHD & \\
\hline Female gender (N, \%) & $26(54.2)$ & $23(51.1)$ & $49(52.7)$ \\
Age (M, SD) & $7.26(0.91)$ & $7.31(0.67)$ & $7.29(0.79)$ \\
FBB-HKS (M, SD) & $1.81(0.60)$ & $0.37(0.54)$ & $1.12(0.92)$ \\
CBCL score (M, SD) & $20.10(10.85)$ & $7.12(7.15)$ & $14.21(11.34)$ \\
\hline
\end{tabular}


[32]. It captures the attachment representations of children between 5 and 9 years. Using small, bendable dolls that represent a family, children are told stories with attachment-relevant content. The experimenter begins the story and asks the child to tell it to the end. The stories are designed to trigger an interaction with the primary caregiver in situations of separation, confrontation, fear, reunion and pain. At a predetermined point in the story, the experimenter asks the child to bring the story to a close. As well as the content, the way in which the child deals with the attachment topics presented is captured by video and subsequently classified [33]. In the SCPDP, the three organized attachment representations secure, insecure-avoidant, insecure-ambivalent as well as disorganized attachment are classified. Next to the categorical determination of these four attachment representations, the derivation of a dimensional attachment security value is additionally possible.

\subsection{German ADHD Rating Scale (FBB-HKS)}

The FBB-HKS is a component of the Diagnostic System for Mental Disorders in Childhood and Adolescence (DISYPS-KJ) [34] and assesses the diagnostic criteria for hyperkinetic disorders from parents or teachers. It includes 20 items of the symptom criteria of both the ICD-10 and DSM-IV as well as additional items assessing symptom onset, symptom duration, pervasiveness, and functional impairment. These criteria are rated on a 4-point answer scale ranging from never (0) to very often (3). The derivation of the three subscales attention, hyperactivity and impulsivity is possible.

\subsection{Child Behavior Checklist 4 - 18 (CBCL/4-18)}

The CBCL/4-18 [35] records psychosocial competencies, behavior problems, emotional problems as well as somatic complaints in children between and including the ages of 4 to 18 years. There are three questionnaires, each with the same content, which can be filled in as self-disclosure by the child or as an external rating by the parents or a teacher. Three competency scales and eight problem behavior scales are considered in the evaluation. From the individual scales, internalizing and externalizing behavior scales as well as a global value for problem behavior are derived.

\section{Statistical Analyses}

All statistical analyses were conducted using SPSS 22 (2013; SPSS: an IBM Company, Chicago, IL). No age difference was found between the group of children with ADHD and the group of children without ADHD. Similarly, the distributions of attachment representations did not differ between boys and girls (see Table 2), neither in the group of children without an ADHD diagnosis $\left(\chi^{2}(2\right.$; $95 \%)=1.97 ; p=0.443)$ nor in the group of children with an ADHD diagnosis $\left(\chi^{2}(3 ; 95 \%)=5.38 ; p=0.123\right)^{1}$.

${ }^{1}$ Due to insufficient expected cell frequencies in some categories, Fisher's exact test was used to determine distribution homogeneity. 
Table 2. Distribution of attachment presentations in children with and without the diagnosis ADHD.

\begin{tabular}{ccccccc}
\hline \multicolumn{5}{c}{ Attachment representations ${ }^{\mathbf{a}}$} \\
\cline { 3 - 6 } & & B & A & C & D & Total \\
\cline { 3 - 6 } & & Secure & Insecure-avoidant & Insecure-ambivalent & Disorganized & \\
\hline No & Girl & $15(65.2)$ & $6(26.1)$ & $0(0.0)$ & $2(8.7)$ & $23(100.0)$ \\
ADHD, & Boy & $11(50.0)$ & $10(45.5)$ & $0(0.0)$ & $1(4.5)$ & $22(100.0)$ \\
N (\%) & Total & $26(57.8)$ & $16(35.6)$ & $0(0.0)$ & $3(6.6)$ & $45(100.0)$ \\
& Girl & $7(26.9)$ & $7(26.9)$ & $3(11.5)$ & $9(34.6)$ & $26(100.0)$ \\
ADHD, & Boy & $3(13.6)$ & $12(54.5)$ & $0(0.0)$ & $7(31.8)$ & $22(100.0)$ \\
N (\%) & Total & $10(20.8)$ & $19(39.6)$ & $3(6.3)$ & $16(33.3)$ & $48(100.0)$ \\
\hline
\end{tabular}

${ }^{a}$ Values from the original data; analyses were performed using the complete imputed and pooled data.

In addition to a descriptive comparison of the distribution of the attachment representations between children with and without ADHD a chi-square test of homogeneity was calculated as statistical verification of the comparison.

A regression model was tested in which attachment representations; externalizing behavior problems as well as their interaction were used as independent variables to account for the variability of the interval-scaled total value of the FBB-HKS. In this regression analysis, the F-values of the individual predictors and their significance are first evaluated in order to assess their influence on the individual independent and dependent variables. A further important statistical measure is the adjusted coefficient of determination $R_{\text {adj }}^{2}$ which depicts the quality of the entire model in so far as it shows the explained portion of variance of a dependent variable using a statistical model.

Data was missing from 13 subjects regarding the continuous ADHD value derived from the assessment of hyperkinetic disorders as well as information from a total of 18 subjects for externalizing behavior problems. For each variable with missing values an imputation model using regression estimators was performed, in-corporating the available information from other variables. To counteract the uncertainty regarding the estimated analysis parameters in the imputation models, a total of five complete data sets were created, in which the imputed values varied in each case. The regression model was applied to each of these data sets to test the second research question. The resulting parameter estimates and estimated standard errors were pooled yielding the sought-after point estimates.

The absence of data could not be attributed to either a characteristic of the group assignment or age or gender. Moreover, Little's MCAR-Test [36], calculated in advance, indicated that the missing values were not systematically connected to other variables $\left(\chi^{2}(2 ; 95 \%)=1.147 ; p=0.564\right)$; thus the requirements for conducting a multiple imputation on the basis of estimates using other variables are met. 


\section{Results}

\subsection{Comparison of Attachment Representations between Children with and without ADHD}

Using the derived FBB-HKS total value, the group with a clinical diagnosis could be very clearly differentiated from the group without a diagnosis: the mean value for the group with a diagnosis $(M=1.81 ; S D=0.60)$ was, with a $T$-value of $T(80)=$ $11.15(p<0.001)$, highly significantly above the mean value for children without a diagnosis $(M=0.37 ; S D=0.54)$. Thus, it can be assumed that the operationalization of a continuous expression of ADHD using the FBB-HKS total value has succeeded.

The descriptive analysis of the distribution of attachment representations between the two groups shows that children with ADHD clearly differ from children without ADHD (Table 2). The distribution of attachment representations in the group of children without an ADHD diagnosis essentially corresponds to the normal population [37], yet the complete absence of insecure-ambivalent representations could not be expected. The distribution of attachment representations in the group of children with an ADHD diagnosis contrasts markedly with this: far fewer children present a secure attachment representation (20.8\%); in contrast, all other categories appear more frequently. The high proportion of disorganized attachment representations (33.3\%) is particularly striking. Statistically, the frequencies differ significantly between the individual categories in terms of the chi-square test of homogeneity with $\chi^{2}(3 ; 95 \%)=19.13(p<0.001)$. Girls and boys do not differ from each other within the diagnosis groups (ADHD vs. no $\mathrm{ADHD})\left(\chi^{2}(3 ; 95 \%)=5.38 ; p<0.123\right.$ and. $\chi^{2}(3 ; 95 \%)=1.97 ; p<0.443$ respectively $)^{1}$.

\subsection{The Relation between Attachment and ADHD}

Since a total of only 3 subjects were classified as insecure-ambivalent, this attachment representation had to be excluded from the analysis of the second research question. The difference between secure and insecure-avoidant attachment within the organized strategies was retained and not subsumed under a further category, because these differences between organized and disorganized as well as between secure and insecure attachment are of particular interest.

In the complete regression model (independent variables: gender, age, attachment, externalizing behavior problems as well as the interaction between attachment and externalizing behavior problems), only the value for externalizing behavior problems proved significant $(F(1,82)=47.40 ; p<0.001)$. The overall quality of the model was $R_{\mathrm{adj}}^{2}=0.45$. The removal of the apparently superfluous interaction did not substantially change the model; externalizing behavior problems remained the single statistically significant predictor $(F(1,82)=46.72 ; p<$ 0.001 ), the adjusted coefficient of determination marginally increased to $R_{\mathrm{adj}}^{2}=$ 0.46 . Attachment, with $F(2,82)=1.55$, proved not to be significant $(p=0.218)$.

In contrast, a simple analysis of variance without controlling for externalizing 
behavior problems revealed a very distinct difference between the attachment representations with regard to the expression of ADHD (Table 3). The overall model was highly significant $(F(2,74)=11.47 ; p<0.001)$. To localize the effect, post-hoc contrasts were calculated to compare secure with insecure as well as organized with disorganized attachment. Both contrasts suggest a highly significant difference $\left(T_{c}=4.12\right.$ and $T_{c}=3.81$; both $\left.p<0.001\right)$.

\section{Discussion}

The aim of this present study was to illuminate on the variations in the distribution of attachment organization in children with and without ADHD. Only those children who presented a simple or unspecified expression of ADHD (predominantly inattentive type) were included in the analysis, in order to be able to provide specific statements about this particular group within the disorder. Moreover, the connection between attachment and ADHD was considered differentially by controlling the conceptually delimitable behavior problems.

The distribution of the attachment representations in children with ADHD differs substantially from those of children without ADHD. The low frequency of the secure attachment representation in these children is equally noticeable as the high occurrence of the disorganized representation. Although a comparison between the attachment representations of children with and without ADHD has been rarely reported, the findings from available literature on the connection between ADHD and attachment nevertheless suggest that these patterns exist in the distribution and correspond to the suppositions formulated in the research questions. The expression of insecure-avoidant strategies as well as the expression of insecure-ambivalent strategies barely differed between the two groups. Comparing distributions of attachment descriptively can of course not imply any causation, yet these findings highlight the role disorganized attachment might play in relation to ADHD. The fact that this attachment representation constitutes one third of the total of attachment representations in the sample of children with $\mathrm{ADHD}$ must lead to the assumption that both constructs are interconnected in a certain way.

Table 3. FBB-HKS values for the various attachment presentations.

\begin{tabular}{ccc}
\hline & \multicolumn{2}{c}{ FBB-HKS total score $^{\mathrm{a}}$} \\
\cline { 2 - 3 } A insecure-avoidant & $N$ & $M(S D)$ \\
B secure & 31 & $1.14(0.89)$ \\
D disorganized & 30 & $0.66(0.80)$ \\
Total & 13 & $1.86(0.63)$ \\
& 77 & $1.23(1.00)$ \\
\hline
\end{tabular}

${ }^{\mathrm{a}}$ Values from the original data; analyses were performed using the complete imputed and pooled data. 
As discussed earlier, controlling for externalizing behavior problems is essential when investigating the connection between attachment and ADHD. Because of their content-related proximity, the high level found for both the F value of the predictor of the externalizing behavior problems as well as the coefficient of determination $\left(R_{\text {adj }}^{2}\right)$ is understandable. However, in this model, no independent effect of attachment on ADHD can be seen. In addition, an interaction between attachment and externalizing behavior problems, which may have been presumed from other studies, did not occur as well. This result contradicts the findings of Thorell et al. [24] and Scholtens et al. [23]. The recognition and classification of our findings is all the more important in the evaluation of the connection between ADHD and attachment. They underline the necessity for developing differentiated hypotheses for specific behavior problems within the symptom conglomerate of ADHD in order to contribute to an expanded understanding of the etiology and the connection to other constructs such as attachment.

Because in our study we only investigated children with the predominantly inattentive type of ADHD, it is highly plausible that the symptom pictures of this specific disorder had a role to play in this conflicting finding. Here, the disparity between the two groups regarding the distribution of attachment representations is completely explained by externalizing behavior problems, which in the mentioned prior studies only constituted a part of the influence. When comparing the expression of the dimensional ADHD value between the individual attachment representations without controlling for externalizing behavior problems, a considerable and highly significant difference is revealed that is in line with the assumed pattern. The expression of ADHD is lowest in securely attached children; in insecure avoidant children somewhat higher; the highest expression, however, is in children with disorganized attachment (Table 3). However, in children with the predominantly inattentive type, the connection between their symptoms and attachment is fully explained by externalizing behaviors; attachment has no independent influence on this.

Despite the disparity in the distribution of attachment representations, the expression of ADHD cannot be attributed to attachment. This constellation suggests that externalizing behavior problems might have an effect on the connection between attachment and ADHD and possibly mediate between them. Baron and Kenny [38] formulated necessary conditions for the presence of mediation, which can be reproduced in the present data: there is a strong connection between predictor (attachment) and mediator (externalizing behavior problems) as well as between mediator and the criterion variable (ADHD). This connection annuls the direct and significant relation between predictor and criterion variable. This observation does not definitively indicate a mediator effect, above all because the mediator model is a causal model that is neither proven in previous studies nor formulated as a hypothesis in this article. It solely attests the fit of our data to a post hoc presumed hypothesis that a complete mediation 
process could be present. A series of other effect mechanisms could equally lead to the same constellation of relations [39] [40]. An explanation could lie in an inverse causal effect in so far as the mediator of the criterion variable, i.e. the externalizing behavior problem is caused by ADHD. Statistically this question remains a dilemma since no causal effect can be derived from the formulated statistical model; only a longitudinal study design could contribute to an explanation.

\section{Limitations}

This study has several limitations. No insecure-ambivalent attachment representations were found in the group of children without ADHD and had to be removed from our analysis. This fact reduces the explanatory power of our findings. In the future, this specific attachment representation should also be analyzed in the context of ADHD to generate findings across the whole range of attachment representations.

The sample consists of children that were available for assessment during the study period and were not randomly selected from a wider population. Furthermore, the study was conducted cross-sectional. To shed more light on the association of attachment and ADHD, there is a strong need for longitudinal as well as prospective studies in this area. A generalization of the findings is thus not appropriate. In addition, the novel findings regarding the absences of a connection between attachment and ADHD should be regarded as tentative pending replication in an independent sample.

\section{Conclusion}

As discussed at the outset, independent effects of attachment on ADHD have been found in previous samples. It can be assumed that this finding is mainly contingent on children with an additional conduct disorder or other undescribed co-morbid symptoms conspicuous in social interactions. In sum, the connection between attachment and ADHD might mostly be indirect because of the overlap with externalizing behavior problems. However, even if attachment is overall a weak predictor, its influence on differentiated aspects of ADHD seems to exist, but the specific types of behavior are not identified, yet. This thesis can only be verified in further studies as the proportion of diverse subgroups of ADHD in the previously described samples cannot be determined. This illustrates the necessity for a detailed sample description regarding the specific characteristics of the ADHD symptom picture. Future studies should also formulate differentiated hypotheses in the investigation of attachment and ADHD as an overall construct.

\section{References}

[1] Kissgen, R. and Franke, S. (2016) An Attachment Research Perspective on ADHD. Neuropsychiatrie, 30, 63-68. https://doi.org/10.1007/s40211-016-0182-1 
[2] Ainsworth, M.D.S., Bell, S.M. and Stayton, D.J. (1974) Infant-Mother Attachment and Social Development: "Socialisation" as a Product of Reciprocal Responsiveness to Signals. In: Richards, P.M., Ed., The Integration of a Child into a Social World, Cambridge University Press, Cambridge, 99-135.

[3] Guttmann-Steinmetz, S., Crowell, J., Doron, G. and Mikulincer, M. (2011) Associations between Mothers' and Children's Secure Base Scripts in ADHD and Community Cohorts. Attachment \& Human Development, 13, 597-610.

https://doi.org/10.1080/14616734.2011.609010

[4] Cassidy, J. (1994) Emotion Regulation: Influences of Attachment Relationships. Monographs of the Society for Research in Child Development, 59, 228-249. https://doi.org/10.2307/1166148

[5] Barkley, R.A. (1997) Behavioral Inhibition, Sustained Attention, and Executive Functions: Constructing a Unifying Theory of ADHD. Psychological Bulletin, 121, 65-94. https://doi.org/10.1037/0033-2909.121.1.65

[6] Olson, S.L. (1996) Developmental Perspectives. In: Sandberg, S., Ed., Hyperactivity Disorders of Childhood. Cambridge Monographs in Child and Adolescent Psychiatry, Cambridge University Press, Cambridge, 149-194.

[7] Teeter, P.A. (2000) Interventions for ADHD: Treatment in Developmental Context. Guilford Press.

[8] Stiefel, I. (1997) Can Disturbance in Attachment Contribute to Attention Deficit Hyperactivity Disorder? A Case Discussion. Clinical Child Psychology and Psychiatry, 2, 45-64. https://doi.org/10.1177/1359104597021005

[9] Clarke, L., Ungerer, J., Chahoud, K., Johnson, S. and Stiefel, I. (2002) Attention Deficit Hyperactivity Disorder Is Associated with Attachment Insecurity. Clinical Child Psychology and Psychiatry, 7, 179-198. https://doi.org/10.1177/1359104502007002006

[10] Matas, L., Arend, R.A. and Sroufe, L.A. (1978) Continuity of Adaptation in the Second Year: The Relationship between Quality of Attachment and Later Competence. Child Development, 49, 547-556. https://doi.org/10.2307/1128221

[11] Fearon, R.M.P. and Belsky, J. (2004) Attachment and Attention: Protection in Relation to Gender and Cumulative Social-Contextual Adversity. Child Development, 75, 1677-1693. https://doi.org/10.1111/j.1467-8624.2004.00809.x

[12] Maslin-Cole, C. and Spieker, S.J. (1990) Attachment as a Basis for Independent Motivation: A View from Risk and Nonrisk Samples. In: Greenberg, M.T., Cicchetti, D. and Cummings, E.M., Eds., Attachment in the Preschool Years: Theory, Research, and Intervention, University of Chicago Press, Chicago, 245-272.

[13] Jacobsen, T., Huss, M., Fendrich, M., Kruesi, M.J. and Ziegenhain, U. (1997) Children's Ability to Delay Gratification: Longitudinal Relations to Mother-Child Attachment. The Journal of Genetic Psychology, 158, 411-426. https://doi.org/10.1080/00221329709596679

[14] Olson, S.L., Bates, J.E. and Bayles, K. (1990) Early Antecedents of Childhood Impulsivity: The Role of Parent-Child Interaction, Cognitive Competence, and Temperament. Journal of Abnormal Child Psychology, 18, 317-334. https://doi.org/10.1007/BF00916568

[15] Barkley, R.A. (2014) Attention-Deficit Hyperactivity Disorder: A Handbook for Diagnosis and Treatment. Guilford Publications.

[16] WHO (2013) Internationale Klassifikation psychischer Störungen: ICD-10 Kapitel V (F) Klinisch-diagnostische Leitlinien. 9. überarb. Aufl. [The International Classi- 
fication of Mental and Behavioural Disorders: ICD-10 Chapter V (F), Clinical Diagnostic Guidelines, 9th Edition.] Verlag Hans Huber, Bern.

[17] American Psychiatric Association (2013) Diagnostic and Statistical Manual of Mental Disorders, 5th Edition: DSM-5. American Psychiatric Publishing, Washington DC.

[18] American Psychiatric Association (2000) Diagnostic and Statistical Manual of Mental Disorders: DSM-IV-TR. American Psychiatric Publishing, Washington DC.

[19] Cassidy, J. and Mohr, J.J. (2001) Unsolvable Fear, Trauma, and Psychopathology: Theory, Research, and Clinical Considerations Related to Disorganized Attachment across the Life Span. Clinical Psychology: Science and Practice, 8, 275-298. https://doi.org/10.1093/clipsy.8.3.275

[20] Van Ijzendoorn, M.H., Schuengel, C. and Bakermans-Kranenburg, M.J. (1999) Disorganized Attachment in Early Childhood: Meta-Analysis of Precursors, Concomitants, and Sequelae. Development and Psychopathology, No. 2, 225-250. https://doi.org/10.1017/S0954579499002035

[21] Bohlin, G., Eninger, L., Brocki, K.C. and Thorell, L.B. (2012) Disorganized Attachment and Inhibitory Capacity: Predicting Externalizing Problem Behaviors. Journal of Abnormal Child Psychology, 40, 449-458. https://doi.org/10.1007/s10802-011-9574-7

[22] Pinto, C., Turton, P., Hughes, P., White, S. and Gillberg, C. (2006) ADHD and Infant Disorganized Attachment: A Prospective Study of Children Next-Born after Stillbirth. Journal of Attention Disorders, 10, 83-91. https://doi.org/10.1177/1087054705286058

[23] Scholtens, S., Rydell, A.-M., Bohlin, G. and Thorell, L.B. (2014) ADHD Symptoms and Attachment Representations: Considering the Role of Conduct Problems, Cognitive Deficits and Narrative Responses in Non-Attachment-Related Story Stems. Journal of Abnormal Child Psychology, 42, 1033-1042.

https://doi.org/10.1007/s10802-014-9854-0

[24] Thorell, L.B., Rydell, A.-M. and Bohlin, G. (2012) Parent-Child Attachment and Executive Functioning in Relation to ADHD Symptoms in Middle Childhood. Attachment \& Human Development, 14, 517-532. https://doi.org/10.1080/14616734.2012.706396

[25] Kissgen, R., Krischer, M., Kummetat, V., Spiess, R., Schleiffer, R. and Sevecke, K. (2009) Attachment Representation in Mothers of Children with Attention Deficit Hyperactivity Disorder. Psychopathology, 42, 201-208. https://doi.org/10.1159/000209333

[26] Goldwyn, R., Stanley, C., Smith, V. and Green, J. (2000) The Manchester Child Attachment Story Task: Relationship with Parental AAI, SAT and Child Behaviour. Attachment \& Human Development, 2, 71-84. https://doi.org/10.1080/146167300361327

[27] Achenbach, T.M. and Edelbrock, C.S. (1981) Behavioral Problems and Competencies Reported by Parents of Normal and Disturbed Children Aged Four through Sixteen. Monographs of the Society for Research in Child Development, 46, 1-82. https://doi.org/10.2307/1165983

[28] Rutter, M., Tizard, J. and Whitmore, K. (1970) Education, Health and Behavior. Longman Group, London.

[29] Pliszka, S.R. (1997) Comorbidity of Attention-Deficit/Hyperactivity Disorder with Psychiatric Disorder: An Overview. The Journal of Clinical Psychiatry, 59, 50-58.

[30] Pillow, D.R., Pelham Jr, W.E., Hoza, B., Molina, B.S. and Stultz, C.H. (1998) Con- 
firmatory Factor Analyses Examining Attention Deficit Hyperactivity Disorder Symptoms and Other Childhood Disruptive Behaviors. Journal of Abnormal Child Psychology, 26, 293-309. https://doi.org/10.1023/A:1022658618368

[31] Gloger-Tippelt, G. and König, L. (2009) Bindung in der mittleren Kindheit: Das Geschichtenergänzungsverfahren zur Bindung 5-bis 8-jähriger Kinder (GEV-B); mit DVD. [Attachment in Middle Childhood: The Story Completion Procedure in Doll play for Children Aged between 5 and 8.] Beltz PVU, Weinheim; Basel.

[32] Bretherton, I., Ridgeway, D. and Cassidy, J. (1990) Assessing Internal Working Models of the Attachment Relationship. In: Greenberg, M.T., Cicchetti, D. and Cummings, E.M., Eds., Attachment in the Preschool Years: Theory, Research, and Intervention, University of Chicago Press, Chicago, 273-308.

[33] Bretherton, I. and Kißgen, R. (2008) Diagnostik der Bindungsqualität im Kindergarten-und Vorschulalter-Die Attachment Story Completion Task (ASCT). [Diagnosticassessment of Attachmentquality in Preschoolers and Kindergartners: The Attachment Story Completion Task (ASCT).] In: Julius, H., Gasteiger-Klicpera, B. and Kißgen, R., Eds., Bindung im Kindesalter: Diagnostik und Interventionen [Attachment in Childhood: Diagnostics and Intervention], Hogrefe, 107-120.

[34] Doepfner, M. and Lehmkuhl, G. (1998) Diagnostic System for Psychiatric Disorders in Children and Adolescents in ICD-10 and DSM IV. Huber, Bern.

[35] Achenbach, T.M. (1991) Manual for the Child Behavior Checklist/4-18 and 1991 Profile. University of Vermont, Dept. of Psychiatry, Burlington.

[36] Little, R.J. (1988) A Test of Missing Completely at Random for Multivariate Data with Missing Values. Journal of the American Statistical Association, 83, 1198-1202. https://doi.org/10.1080/01621459.1988.10478722

[37] Van IJzendoorn, M.H. and Kroonenberg, P.M. (1988) Cross-Cultural Patterns of Attachment: A Meta-Analysis of the Strange Situation. Child Development, 147-156. https://doi.org/10.1111/j.1467-8624.1988.tb03202.x

[38] Baron, R.M. and Kenny, D.A. (1986) The Moderator-Mediator Variable Distinction in Social Psychological Research: Conceptual, Strategic, and Statistical Considerations. Journal of Personality and Social Psychology, 51, 1173-1182. https://doi.org/10.1037/0022-3514.51.6.1173

[39] Fiedler, K., Schott, M. and Meiser, T. (2011) What Mediation Analysis Can (Not) Do. Journal of Experimental Social Psychology, 47, 1231-1236.

[40] Judd, C.M. and Kenny, D.A. (2010) Data Analysis in Social Psychology: Recent and Recurring Issues. Handbook of Social Psychology. 